\title{
A ZADOFF-CHU MATRIX TRANSFORM PRECODING BASED LOCALIZED-OFDMA UPLINK SYSTEM: A PAPR ANALYSIS WITH RRC PULSE SHAPING
}

\author{
Imran BAIG, Varun JEOTI \\ Electrical and Electronic Engineering Department, Universiti Teknologi PETRONAS, Tronoh, 31750, Perak, Malaysia, \\ e-mail: imran_baig_mirza@yahoo.com, varun_jeoti@petronas.com.my
}

\begin{abstract}
High peak-to-average power ratio (PAPR) is one of the major drawbacks in the orthogonal frequency division multiple access (OFDMA) systems. In this paper, we present a zadoff-chu matrix transform (ZCMT) precoding based localized OFDMA uplink system with improved PAPR. The proposed system is based on precoding the constellation symbols with ZCMT precoder. Extensive computer simulations have been performed to analyze the PAPR of the proposed system with the root-raised-cosine (RRC) pulse shaping. Simulation results show that, the proposed system has better PAPR gain than the hadamard-transform (WHT) precoded Localized-OFDMA uplink systems and the conventional Localized-OFDMA uplink systems.
\end{abstract}

Keywords: Peak-to-average power ratio (PAPR), Orthogonal frequency division multiple access (OFDMA), Zadoff-chu matrix transform (ZCMT), Localized-OFDMA uplink, Hadamard transform (WHT), Root-raised-cosine (RRC)

\section{INTRODUCTION}

Orthogonal frequency division multiple access (OFDMA) is a multiple access version of the orthogonalfrequency-division-multiplexing (OFDM) system. The OFDMA system splits the high speed data stream into a number of parallel low data rate streams and these low rates data streams are transmitted simultaneously over a number of orthogonal subcarriers. The key difference between the OFDM and the OFDMA is that instead of being allocated all of the available subcarriers the base station assigns a subset of carriers to each user in order to accommodate the several transmissions at the same time. An inherent gain of OFDMA is its ability to exploit the multiuser-diversity through subchannel allocation. Additionally, OFDMA has the advantage of simple decoding at the receiver side due to the absence of intercarrier-interference (ICI). Other benefit of OFDMA includes better granularity and improved link budget in the uplink communications [1]-[2].

There are two different approaches to do subcarrier mapping in OFDMA system, localized subcarrier mapping and distributed subcarrier mapping. Distributed subcarrier mapping can be further divided in to two modes i.e. interleaved mode and random interleaved mode. To avoid the ICI in the uplink due to the doppler frequency shift, the localized OFDMA is more preferred for practical implementations than the interleaved OFDMA and random interleaved OFDMA. Fig. 1 shows the subcarrier mapping in interleaved mode, where the subcarriers are mapped equidistant to each other's. Fig. 2, further explain the concept of localized subcarrier mapping, where the subcarrier mapping is done in adjacent.

OFDMA is now widely adopted in the various communication standards like the worldwide interoperability for microwave access (WiMAX), the mobile broadband wireless access (MBWA), the evolved UMTS terrestrial radio access (E-UTRA) and the ultra mobile broadband (UMB). OFDMA is also a strong candidate for the wireless regional area networks (WRAN) and the long term evolution advanced (LTE-A).

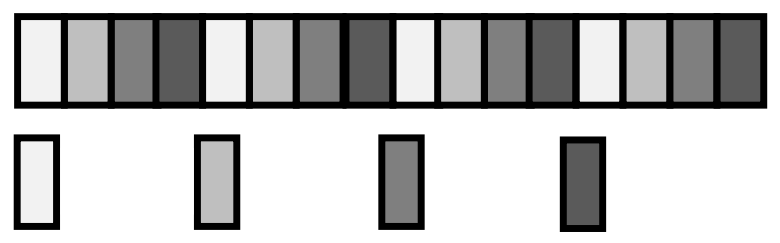

Fig. 1 Interleaved OFDMA

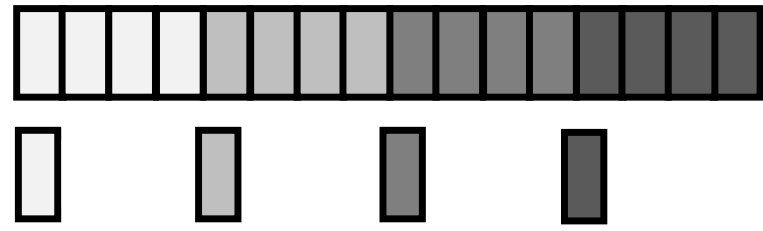

Fig. 2 Localized OFDMA

However, the OFDMA has some drawbacks, among others the peak-to-average power ratio (PAPR) is still one of the major drawbacks in the transmitted OFDMA signals [3]. The nonlinear amplification in the OFDMA transmission systems has a crucial influence on the overall performance and therefore its effects must be taken into account very carefully [4]. Therefore, for zero distortion of the OFDMA signal the high-power-amplifier (HPA) must not only operate in its linear region but also with sufficient back-off. Thus, HPA with a large dynamic range is required for OFDMA systems. These amplifiers are very expensive and are major cost component of the OFDMA systems. Thus, if we reduce the PAPR it not only means that we are reducing the cost of the OFDMA systems and reducing the complexity of analog-to-digital (A/D) and digital-to-analog (D/A) converters but also increasing the transmit power thus, for same range improving received signal-to-noise ratio (SNR) or for the same SNR improving range.

A large number of PAPR reduction techniques have been proposed in the literature. Among them, schemes like phase optimization [5], constellation shaping [6], selective-mapping (SLM) [7], nonlinear companding transforms [8], tone-reservation (TR), tone-injection (TI) 
[9]-[10], partial-transmit-sequence (PTS) [11], clipping and filtering [12]-[13 ], precoding based techniques [14][17] precoding based selected mapping (PSLM) techniques [18]-[19] and phase modulation transform [20]-[21] are popular. The precoding based techniques shows great promise as they are simple linear techniques to implement without the need of any side information.

This paper presents a zadoff-chu matrix transform (ZCMT) precoding based localized OFDMA uplink system for PAPR reduction. For the PAPR analysis of the proposed system, the famous root-raised-cosine (RRC) pulse shaping is implemented. This paper is organized as follows: Section 2 describes the basics of the localized OFDMA uplink systems, In Section 3 we present the proposed system with improved PAPR, Section 4 presents the computer simulation results and section 5 concludes the paper.

\section{LOCALIZED-OFDMA UPLINK SYSTEM}

Fig. 3 illustrates the block diagram of the localized OFDMA uplink systems, where the subcarrier mapping is done in adjacent. In the localized OFDMA uplink systems the baseband modulated symbols are passed through serial-to-parallel $(\mathrm{S} / \mathrm{P})$ converter which generates the complex vector of size $L$. We can write the complex

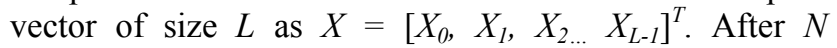
subcarrier mapping in the localized mode to the $X$, we get $\hat{X}_{l}=\left[\hat{X}_{0}, \hat{X}_{1}, \ldots \hat{X}_{N-1}\right]^{T}$. The complex baseband localized OFDMA uplink signal with $N$ system subcarriers and $L$ user subcarriers can be written as:

$x_{n}^{(k)}=\frac{1}{\sqrt{N}} \sum_{l=0}^{L-1}\left(\hat{X}_{l}^{(k)} \cdot e^{j 2 \pi \frac{(k L+l)}{N} n}\right)$

where, $\hat{X}_{l}$ we get after subcarrier mapping, $j=\sqrt{-1}$ and $n=$ $0,1,2 \ldots N-1 . \hat{X}_{l}^{(k)}$ is modulated signal on subcarrier $l$ for the $k^{\text {th }}$ user with users index $k=1,2, \ldots, Q-1$.

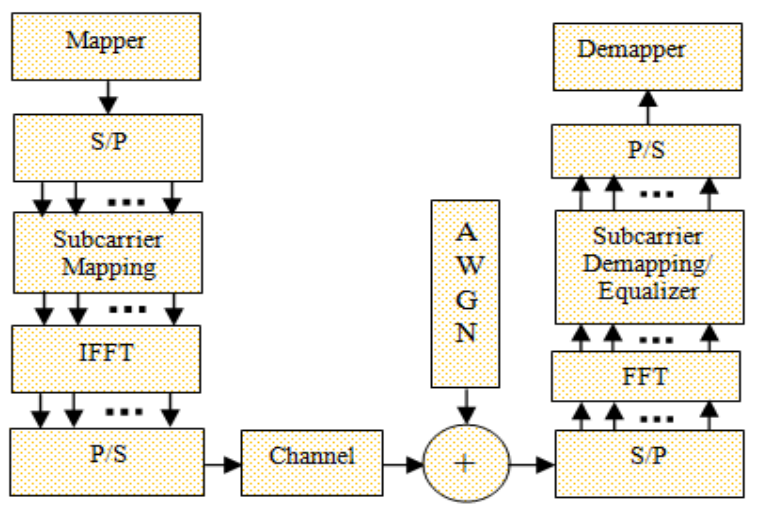

Fig. 3 Localized-OFDMA Uplink system

\section{PROPOSED MODEL}

\subsection{Zadoff-Chu (ZC) sequences and Zadoff-Chu Matrix Transform (ZCMT)}

The zadoff-chu (ZC) sequences are class of poly-phase sequences having optimum correlation properties. ZC sequences have an ideal periodic autocorrelation and the constant magnitude. According to [22]-[23] ZC sequences of length $L$ can be defined as:

$a_{n}= \begin{cases}e^{\frac{j 2 \pi r}{L}\left(\frac{k^{2}}{2}+q k\right)} & \text { for L Even } \\ e^{\frac{j 2 \pi r}{L}\left(\frac{k(k+1)}{2}+q k\right)} \cdot & \text { for L Odd }\end{cases}$

where, $k=0,1,2 \ldots L-1, q$ is any integer and $r$ is any integer relatively prime to $L$. The kernel of the ZCMT is defined in (3). For $N=L \times L$ and $j=\sqrt{-1}$, the ZCMT $A$ of size $N=L \times L=L^{2}$ is obtained by reshaping the $\mathrm{ZC}$ sequence by $k=m L+l$ as hereunder:

$A=\left[\begin{array}{cccc}a_{00} & a_{01} & \ldots & a_{0(L-1)} \\ a_{10} & a_{11} & \ldots & a_{1(L-1)} \\ \vdots & \vdots & \ddots & \vdots \\ a_{(L-1) 0} & a_{(L-1) 1} & \ldots & a_{(L-1)(L-1)}\end{array}\right]$

Here $m$ is the row variable and $l$ the column variable. In other words, the $L^{2}$ point long $\mathrm{ZC}$ sequence fills the kernel of the matrix transform row-wise.

\subsection{ZCMT Precoding Based Localized-OFDMA Uplink System}

Fig. 4 shows the ZCMT precoding based localized OFDMA uplink system. In this system a precoding matrix $A$ of dimension $N=L \times L$ is applied to the constellation symbols before the subcarrier mapping and the IFFT to reduce the PAPR.

In the ZCMT precoding based localized OFDMA uplink system baseband modulated data is passed through $\mathrm{S} / \mathrm{P}$ convertor which generates the complex vector of size $L$ that can be written as $X=\left[\begin{array}{llll}X_{0} & X_{1} & \ldots & X_{L-1}\end{array}\right]^{\mathrm{T}}$. Then the ZCMT precoding is applied to this complex vector which transforms this complex vector into new vector of length $L$ that can be written as $Y=A X=\left[Y_{0}, Y_{l}, Y_{2 \ldots} Y_{L-1}\right]^{T}$, where $A$ is a precoder matrix of size $N=L \times L$ and $Y_{m}$ can be written as:

$Y_{m}=\sum_{l=0}^{L-1} a_{m, l} . X_{l} \quad m=0,1, \ldots L-1$

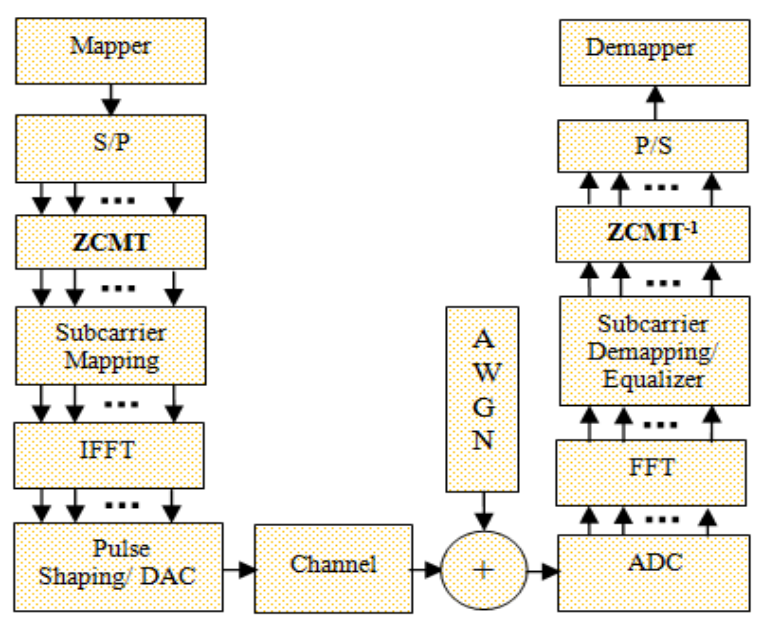

Fig. 4 ZCMT Precoding based Localized-OFDMA uplink system 
$a_{m, l}$ means $m^{\text {th }}$ row and $l^{\text {th }}$ column of precoder matrix. Expanding equation (4) using the row-wise sequence reshaping $k=m L+l$ and putting $q=0, r=1$ in the equation (2) we get:

$$
\begin{aligned}
Y_{m} & =\sum_{l=0}^{L-1}\left(e^{j \frac{\pi(m L+l)^{2}}{L^{2}}}\right) \cdot X_{l} \\
& =e^{j \pi m^{2}} \sum_{l=0}^{L-1}\left(\left(e^{j \frac{\pi l^{2}}{L^{2}}} \cdot X_{l}\right) \cdot e^{j \frac{2 \pi m l}{L}}\right)
\end{aligned}
$$

where $m=0,1,2 \ldots L-1$.

The equation (6) represents the ZCMT precoded data/constellations symbols or signal. Mathematically, the subcarrier mapping in localized mode for the ZCMT precoding based localized OFDMA system can be done as:

$$
\widehat{Y}_{l}= \begin{cases}Y_{m} & 0 \leq m \leq L-1 \\ 0 & L \leq m \leq N-1\end{cases}
$$

where $N$ : System subcarriers, $L$ : User subcarriers, $Q$ : Subchannels/Users $(Q=N / L)$.

The subchannel $(q)$ is composed of subcarriers with index set $\{(q L),(q L+1),(q L+2) \ldots(q L+L-1)\}$, where $q=0,1,2 \ldots Q-1$. Suppose the $k^{\text {th }}$ user is assigned to subchannel $(k)$ then the complex baseband ZCMT precoded localized OFDMA uplink signal for $k^{\text {th }}$ user can be written as:

$x_{n}^{(k)}=\frac{1}{\sqrt{N}} \sum_{l=0}^{L-1}\left(\hat{Y}_{l}^{(k)} \cdot e^{j 2 \pi \frac{(k L+l)}{N} n}\right), n=0,1 \ldots N-1$

where, $\hat{Y}_{l}^{(k)}$ is modulated signal on subcarrier $l$ for $k^{\text {th }}$ user. The complex passband signal of the ZCMT precoded localized OFDMA uplink after RRC pulse shaping can be written as:

$$
x(t)=e^{j \omega_{c} t} \sum_{n=0}^{N-1} x_{n}^{(k)} \cdot r(t-n \check{T})
$$

Where $\omega_{c}$ is carrier frequency, $r(t)$ is baseband pulse and $\check{T}=\left(\frac{M}{N}\right) . T$ is compressed symbol duration after IFFT and $T$ is symbol duration is seconds. The RRC pulse shaping filter can be defined as:

$$
r(t)=\frac{\sin \left(\frac{\pi t}{\widetilde{T}}(1-\alpha)\right)+4 \alpha \frac{t}{\widetilde{T}} \cdot \cos \left(\frac{\pi t}{\widetilde{T}}(1+\alpha)\right)}{\frac{\pi t}{\widetilde{T}}\left(1-\frac{16 \alpha^{2} t^{2}}{\widetilde{T}^{2}}\right)}
$$

where rolloff factor $\alpha$ is $0 \leq \alpha \leq 1$. The PAPR of the ZCMT precoded localized OFDMA uplink signals in (9) with RRC pulse shaping can be written as:

$$
P A P R=\frac{\max _{0 \leq t \leq N \widetilde{T}}|x(t)|^{2}}{\frac{1}{N \widetilde{T}} \int_{0}^{N \widetilde{T}}|x(t)|^{2} d t}
$$

The PAPR of the ZCMT precoded localized OFDMA uplink signals in (8) without pulse shaping can be written as:

$P A P R=\frac{\max _{n=0,1, \ldots, N-1}\left|x_{n}^{(k)}\right|^{2}}{\frac{1}{M} \sum_{n=0}^{N-1}\left|x_{n}^{(k)}\right|^{2}}$
It should be pointed out that the orthogonality of the symbols after introducing precoding is maintained, as the precoding matrix is cyclic auto-orthogonal [20].

\section{SIMULATION RESULTS}

Extensive simulations in $\operatorname{MATLAB}^{(\mathrm{R})}$ have been performed to evaluate the performance of the proposed ZCMT precoded localized OFDMA uplink system with and without pulse shaping. To show the PAPR analysis of the proposed system, the data is generated randomly then modulated by QPSK, 16-QAM and 64-QAM respectively. We evaluate the PAPR statistically by using complementary-cumulative-distribution function (CCDF). The CCDF of the PAPR for the ZCMT precoded localized OFDMA uplink signals is used to express the probability of exceeding a given threshold $\mathrm{PAPR}_{0} \quad(C C D F=$ Prob $\left.\left(P A P R>P A P R_{0}\right)\right)$. We also compare the PAPR results of the proposed system with the hadamard transform (WHT) precoded localized OFDMA uplink systems and the conventional localized OFDMA uplink systems respectively. To show the PAPR analysis of the proposed system with RRC pulse shaping in the MATLAB $^{\circledR}$ we considered RRC rolloff factor $\alpha=0.22$. All the simulations have been performed based on the $10^{5}$ random data blocks. Simulation parameters that we use are given in the following Table 1 .

Table 1 System Parameters

\begin{tabular}{|l|l|}
\hline Channel Bandwidth & $5 \mathrm{MHz}$ \\
\hline Oversampling Factor & 4 \\
\hline User Subcarriers & 64 \\
\hline System Subcarriers & 256 \\
\hline Precoding & WHT and ZCMT \\
\hline Modulation & QPSK, 16-QAM, 64-QAM \\
\hline Pulse Shaping & Root Raised Cosine (RRC) \\
\hline Typical RRC Roll-Off Factor & $\alpha=0.22$ \\
\hline Subcarrier Mapping Mode & Localized \\
\hline CCDF Clip Rate & $10^{-3}$ \\
\hline
\end{tabular}

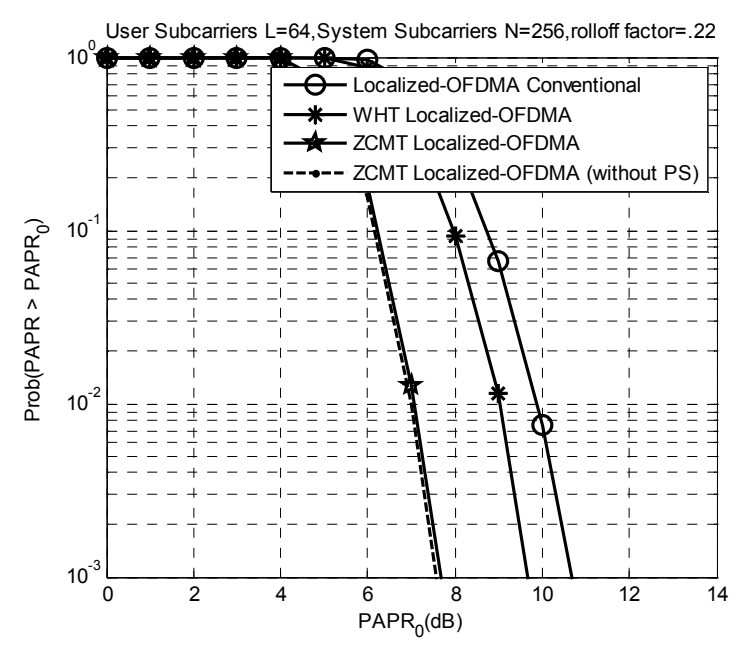

Fig. 5 CCDF Comparison of PAPR of the ZCMT precoded Localized-OFDMA uplink system with pulse shaping and ZCMT precoded Localized-OFDMA uplink system without pulse shaping, with the WHT precoded Localized-OFDMA uplink system and the original Localized-OFDMA uplink system for QPSK modulation 
Fig. 5 shows the CCDF comparison of the PAPR for the ZCMT precoded localized OFDMA uplink system with RRC pulse shaping and the ZCMT precoded localized OFDMA uplink system without pulse shaping with the WHT precoded localized OFDMA uplink systems and the conventional localized OFDMA uplink systems. At the clip rate of $10^{-3}$, with user subcarriers $L=64$ and system subcarriers $N=256$, the PAPR is $10.8 \mathrm{~dB}, 9.7 \mathrm{~dB}, 7.8 \mathrm{~dB}$, and $7.7 \mathrm{~dB}$ respectively, for the conventional localized OFDMA uplink systems, the WHT precoded localized OFDMA uplink systems, the ZCMT precoded localized OFDMA uplink system using RRC pulse shaping and the ZCMT precoded localized OFDMA uplink system without pulse shaping for QPSK modulation.

Fig. 6 shows the CCDF comparison of the PAPR for the ZCMT precoded localized OFDMA uplink system with RRC pulse shaping and the ZCMT precoded localized OFDMA uplink system without pulse shaping with the WHT precoded localized OFDMA uplink systems and the conventional localized OFDMA uplink systems. At the clip rate of $10^{-3}$, with user subcarriers $L=64$ and system subcarriers $N=256$ the PAPR is 10.7 $\mathrm{dB}, 10 \mathrm{~dB}, 8.4 \mathrm{~dB}$ and $8.4 \mathrm{~dB}$ respectively, for the conventional localized OFDMA uplink systems, the WHT precoded localized OFDMA uplink systems, the ZCMT precoded localized OFDMA uplink system using RRC pulse shaping and the ZCMT precoded localized OFDMA uplink system without pulse shaping for 16-QAM modulation.

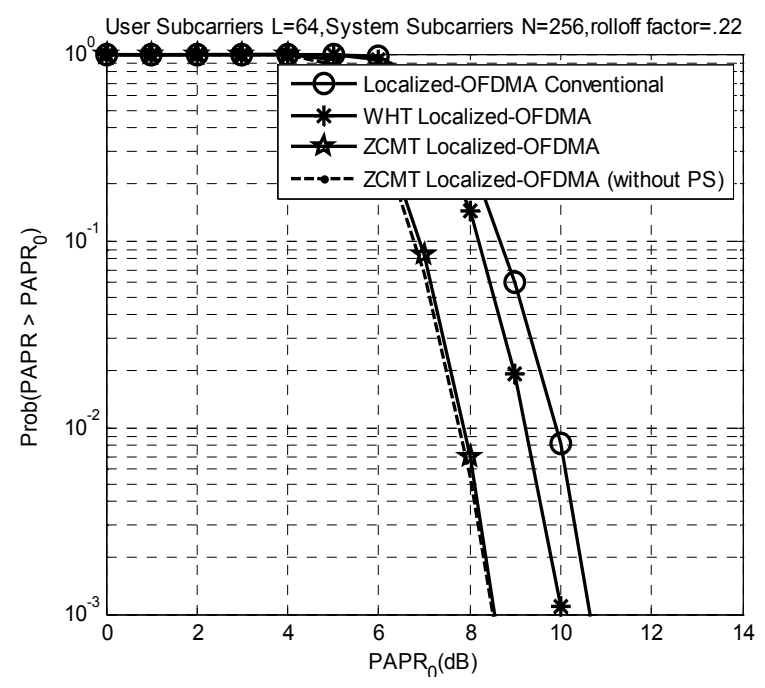

Fig. 6 CCDF Comparison of PAPR of the ZCMT precoded Localized-OFDMA uplink system with pulse shaping and ZCMT precoded Localized-OFDMA uplink system without pulse shaping, with the WHT precoded Localized-OFDMA uplink system and the original Localized-OFDMA uplink system for 16-QAM modulation

Fig. 7 shows the CCDF comparison of the PAPR for the ZCMT precoded localized OFDMA uplink system with RRC pulse shaping and the ZCMT precoded localized OFDMA uplink system without pulse shaping with the WHT precoded localized OFDMA uplink systems and the conventional localized OFDMA uplink systems. At the clip rate of $10^{-3}$, with user subcarriers
$L=64$ and system subcarriers $N=256$ the PAPR is 10.7 $\mathrm{dB}, 10 \mathrm{~dB}, 8.7 \mathrm{~dB}$ and $8.6 \mathrm{~dB}$ respectively, for the conventional localized OFDMA uplink systems, the WHT precoded localized OFDMA uplink systems, the ZCMT precoded localized OFDMA uplink system using RRC pulse shaping and ZCMT precoded localized OFDMA uplink system without pulse shaping for 64-QAM modulation.

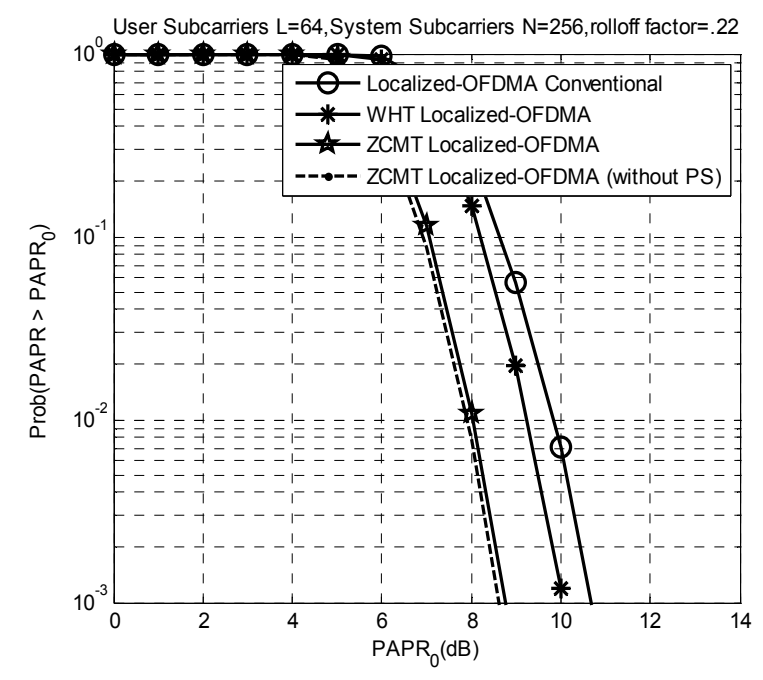

Fig. 7 CCDF Comparison of PAPR of the ZCMT precoded Localized-OFDMA uplink system with pulse shaping and ZCMT precoded Localized-OFDMA uplink system without pulse shaping, with the WHT precoded Localized-OFDMA uplink system and the original Localized-OFDMA uplink system for 64-QAM modulation

Table 2 summarizes the PAPR of the conventional localized OFDMA uplink systems, the WHT localized OFDMA uplink systems, the ZCMT localized OFDMA uplink system, using RRC pulse shaping and the ZCMT localized OFDMA uplink system without pulse shaping respectively, for QPSK, 16-QAM and 64-QAM modulation. From Table 2 it is concluded that, the ZCMT precoded localized OFDMA uplink system with or without pulse shaping has low PAPR than the WHT precoded localized OFDMA uplink systems and the conventional localized OFDMA uplink systems.

Table 2 At the clip rate of $10^{-3}$, the PAPR comparisons of Localized-OFDMA uplink, WHT Localized-OFDMA uplink, ZCMT Localized-OFDMA uplink using RRC pulse shaping (roll off factor $\alpha=0.22$ ) and ZCMT Localized-OFDMA uplink without pulse shaping, for $L=64$ and $N=256$

\begin{tabular}{|l|c|c|c|}
\hline \multirow{2}{*}{$\begin{array}{l}\text { Uplink Transmission } \\
\text { Scheme }\end{array}$} & \multicolumn{3}{|c|}{ PAPR } \\
\cline { 2 - 4 } & QPSK & 16-QAM & 64-QAM \\
\hline Localized-OFDMA & $10.8 \mathrm{~dB}$ & $10.7 \mathrm{~dB}$ & $10.7 \mathrm{~dB}$ \\
\hline $\begin{array}{l}\text { WHT Localized- } \\
\text { OFDMA }\end{array}$ & $9.7 \mathrm{~dB}$ & $10.0 \mathrm{~dB}$ & $10.0 \mathrm{~dB}$ \\
\hline $\begin{array}{l}\text { ZCMT Localized- } \\
\text { OFDMA }\end{array}$ & $7.8 \mathrm{~dB}$ & $8.4 \mathrm{~dB}$ & $8.7 \mathrm{~dB}$ \\
\hline $\begin{array}{l}\text { ZCMT Localized- } \\
\begin{array}{l}\text { OFDMA without RRC } \\
\text { Pulse Shaping }\end{array}\end{array}$ & $7.7 \mathrm{~dB}$ & $8.4 \mathrm{~dB}$ & $8.6 \mathrm{~dB}$ \\
\hline
\end{tabular}




\section{CONCLUSIONS}

In this paper, a ZCMT precoded localized OFDMA uplink system has been proposed for the PAPR reduction. Computer simulation shows that the PAPR of the proposed system is less than the WHT precoded localized OFDMA uplink systems and the conventional localized OFDMA uplink systems. Proposed system is efficient, signal independent, distortionless and do not require any complex optimizations. Additionally, ZCMT precoded localized OFDMA uplink system also take the advantage of the frequency variations of the communication channel and can also offer substantial performance gain in fading multipath channels. Thus, it is concluded that the ZCMT precoded localized OFDMA uplink system is more favourable than the WHT precoded localized OFDMA systems and conventional localized OFDMA uplink systems.

\section{REFERENCES}

[1] KNOPP, R. - HUMBLET, P. A.: Information Capacity and Power Control in Single-Cell Multiuser Communications, IEEE International conference on Communications, Seattle, WA, vol. 1, pp. 331-335, June 1995.

[2] TSE, D.: Optimal Power Allocation over Parallel Gaussian Broadcast Channels, International Symposium on Information theory, Ulm, Germany, pp. 27, June 1997.

[3] WANG, H. - CHEN, B.: Asymptotic Distributions and Peak Power Analysis for Uplink OFDMA Signals, IEEE International Conference on Acoustics, Speech, and Signal Processing, vol. 4, pp. IV, May 2004.

[4] GAZDA, J. et al.: Joint Evaluation of Nonlinear Distortion Effects and Signal Metrics in OFDM based Transmission Systems, Acta Electrotechnica et Informatica, vol. 9, no. 4, pp. 55-60, 2009.

[5] NIKOOKAR, H. - LIDSHEIM, K. S.: Random Phase Updating Algorithm for OFDM Transmission with Low PAPR, IEEE Trans. Broadcasting, vol. 48, no. 2, pp. 123-128, June 2002.

[6] KOU, Y. - LU, W. - ANTONIOU, A.: A New Peak-to-Average Power-Ratio Reduction Algorithm for OFDM Systems via Constellation Extension, IEEE Trans. Wireless Communications, vol. 6, no. 5, pp. 1823-1832, May 2007.

[7] LIM, D. W. et al.: A New SLM OFDM Scheme with Low Complexity for PAPR Reduction, IEEE Signal Processing Letters, vol. 12, no. 2, pp. 93-96, Feb. 2005.

[8] JIANG, T. et al.: Two Novel Nonlinear Companding Schemes with Iterative Receiver to Reduce PAPR in Multicarrier Modulation Systems, IEEE Trans. Broadcasting, vol. 52, no. 2, pp. 268-273, Mar. 2006.
[9] MOURELO, J. T.: Peak to Average Power Ratio Reduction for Multicarrier Modulation, PhD thesis, University of Stanford, Stanford, 1999.

[10] YOO, S. et al.: A Novel PAPR Reduction Scheme for OFDM Systems: Selective Mapping of Partial Tones (SMOPT), IEEE Trans. Consumer Electronics, vol. 52, no. 1, pp. 40-43, Feb. 2006.

[11] HAN, S. H. - LEE, J. H.: PAPR Reduction of OFDM Signals Using a Reduced Complexity PTS Technique, IEEE Signal Processing Letters, vol. 11, Iss. 11, pp. 887-890, Nov. 2004.

[12] WANG, L. - TELlAMBURA, C.: A Simplified Clipping and Filtering Technique for PAR Reduction in OFDM Systems, IEEE Signal Processing Letters, vol. 12, no. 6, pp. 453-456, June 2005.

[13] URBAN, J. - MARŠÁLEK, R.: The Combination of Clipping and Filtering with Selective Mapping Methods for Peak to Average Power Ratio Reduction in OFDM, Acta Electrotechnica et Informatica, vol. 9, no. 4, pp. 24-29, 2009.

[14] MIN, Y. K. - JEOTI, V.: A Novel Signal Independent Technique for PAPR Reduction in OFDM Systems, International Conference on Signal Processing, Communications and Networking (ICSCN), Chennai, India, pp. 308-311, 22-24 Feb. 2007.

[15] BAIG, I. - JEOTI, V.: Novel Precoding Based PAPR Reduction Techniques for Localized-OFDMA Uplink System of LTE-Advanced, Journal of Telecommunication, Electronic and Computer Engineering (JTEC), vol. 2, no. 1, pp. 49-58, 2010.

[16] BAIG, I. - JEOTI, V.: PAPR Analysis of DHTPrecoded OFDM System for M-QAM, International Conference on Intelligent and Advanced Systems, (ICIAS), Kuala Lumpur, Malaysia, pp. 1-4, 15-17 June 2010.

[17] BAIG, I. - JEOTI, V.: PAPR Reduction in OFDM Systems: Zadoff-Chu Matrix Transform Based Pre/Post-Coding Techniques, 2nd International Conference on Computational Intelligence, Communication Systems and Networks, (CICSyN), Liverpool, UK, pp. 373-377, 28-30 July 2010.

[18] JEOTI, V. - BAIG, I.: A Novel Zadoff-Chu Precoder Based SLM Technique for PAPR Reduction in OFDM Systems, invited paper, Proceedings of 2009 IEEE International Conference on Antennas, Propagation and Systems, Johor, Malaysia, 3-5 Dec. 2009.

[19] BAIG, I. - JEOTI, V.: DCT Precoded SLM Technique for PAPR Reduction in OFDM Systems, International Conference on Intelligent and Advanced Systems, (ICIAS), Kuala Lumpur, Malaysia, pp. 1-6, 15-17 June 2010. 
[20] TASI, Y. - ZHANG, G. - WANG, X.: Orthogonal Polyphase Codes for Constant Envelope OFDMCDMA System, IEEE, WCNC, Las Vegas, NV, pp. 1396-1401, 3-6 Apr. 2006.

[21] THOMPSON, S. C. - AHMED, A. U. - PROAKIS, J. G. - ZEIDLER, J. R. - GEILE, M. J.: "Constant Envelope OFDM”, IEEE Trans. Communications, vol. 56, pp. 1300-1312, Aug. 2008.

[22] CHU, D. C.: "Polyphase Codes with Good Periodic Correlation Properties", IEEE Trans. Inform. Theory, vol. IT-18, pp. 531-532, July 1972.

[23] POPOVIC, B. M.: "Spreading Sequences for MultiCarrier CDMA Systems", In IEE Colloquium CDMA Techniques and Applications for Third Generation Mobile Systems, London, pp. 8/1-8/6, May 19, 1997.

Received November 21, 2010, accepted April 5, 2011

\section{BIOGRAPHIES}

Imran Baig received his MSc Computer Engineering degree from Centre for Advanced Studies in Engineering (CASE), Islamabad, Pakistan in 2005. In Feb. 2005 he joined Federal Urdu University of Art, Science and Technology, Islamabad Pakistan as Computer Science Lecture. In Feb. 2009 he moved to Universiti Teknologi PETRONAS, Malaysia for PhD-EE. His research interests include PAPR reduction in OFDM systems, internet security, wireless ad hoc and sensor networks and 4G networks and statistical signal processing.

Varun Jeoti received his $\mathrm{PhD}$ degree from Indian Institute of Technology (IIT), Delhi, India, in 1992. He worked on several sponsored R\&D projects in IIT Delhi and IIT Madras during 1980 to 1989 developing Surface Acoustic Wave Pulse Compression filters, underwater optical receivers etc. He was a Visiting Faculty in Electronics department in Madras Institute of Technology for about 1 year during 1989 to 1990 and joined Delhi Institute of Technology (presently called Netaji Subhash Institute of Technology) for next 5 years till 1995. He moved to Electrical \& Electronic Engineering (E\&E Engg) department of Universiti Sains Malaysia in 1995 and joined E\&E Engg department of Universiti Teknologi PETRONAS in 2001 where he leads the Advanced Communication Engineering cluster. His research interests are in signal processing and wireless communication relating to wireless LAN and MAN technologies, LTE, cognitive radio, maritime communication, distributed video coding and security. 